\title{
UM MODELO PARA GERENCIA DE CONHECIMENTO EM PROJETO DE SOFTWARE BASEADO NO FLUXO DE CONHECIMENTO
}

\author{
Plácido Antônio de Souza Neto \\ Centro Federal de Educação Tecnológica do Rio Grande do Norte (CEFET-RN) \\ Caixa Postal 1.559 - 59.015-000 - Natal - RN - Brasil \\ placidoneto@cefetrn.br \\ João Maria Filgueira \\ Centro Federal de Educação Tecnológica do Rio Grande do Norte (CEFET-RN) \\ Caixa Postal 1.559 - 59.015-000 - Natal - RN - Brasil \\ imfilgueira@cefetrn.br
}

\begin{abstract}
RESUMO
Este trabalho apresenta o KFM, Knowledge Flow Model, um modelo de fluxo de conhecimento na aplicação e utilização em desenvolvimento de sistemas e ambientes de pesquisa. Para a construção desse modelo, são aplicados conceitos de gerência de conhecimento em ambientes de software. Tais conceitos têm como objetivo a melhoria do uso do conhecimento em ambientes de pesquisa e desenvolvimento. Conceitos e exemplos da aplicação de gerência de conhecimento também são tratados nesse trabalho. Após a integração desses conceitos, um modelo é proposto para que seja utilizado e testado na área de desenvolvimento de software. $O$ trabalho também apresenta um sistema para aplicação específica em acompanhamento de projetos de desenvolvimento de software.
\end{abstract}

PALAVRAS CHAVE: modelo de conhecimento, projeto de software, ambiente de desenvolvimento, processo de gerência, desenvolvimento de software.

\section{A KNOWLEDGE MANAGEMENT'S MODEL IN SOFTWARE PROJECT BASED ON THE KNOWLEDGE FLOW}

\begin{abstract}
This work presents the KFM, Knowledge Flow Model, a knowledge flow model to application and use in systems development and research environments. For the construction of this model, concepts of knowledge management in software environments are applied. Such concepts have as objectives the knowledge use improvement in research and development's environments. The concepts and examples of knowledge management application also are treated in this work. After integration of these concepts, a model is proposed to be used and tested in the software development area. This work also presents a specific application system to accompaniment of development software projects.
\end{abstract}

KEYWORDS: knowledge model, software project, research environment, management process, software development.

\section{UM MODELO PARA GERÊNCIA DE CONHECIMENTO EM PROJETO DE SOFTWARE BASEADO NO FLUXO DE CONHECIMENTO}




\section{Introdução}

O conhecimento obtido no desenvolvimento de software é um recurso importante e o seu uso promove um aprendizado evolutivo, evitando que um mesmo erro seja cometido novamente. Porém, para atingir esse aprendizado, é necessário que o conhecimento esteja disponível e acessível. Neste contexto, ambientes de gerência de conhecimento podem ser bastante úteis. A gerência de conhecimento facilita a criação, acesso e reuso do conhecimento e seu objetivo principal é promover o surgimento de conhecimento novo, seu armazenamento e compartilhamento.

Embora cada projeto de desenvolvimento de software seja único, experiências similares e comuns a eles podem ajudar aos desenvolvedores na execução de suas atividades. $\mathrm{O}$ reuso desse conhecimento ajuda a evitar que falhas se repitam e auxilia na solução de problemas recorrentes.

Mas, para ser efetiva, a gerência de conhecimento deve ser integrada ao processo de trabalho. Assim, a gerência de conhecimento em ambientes de software irá auxiliar o desenvolvimento continuo e um crescente aprendizado do projeto de software.

$\mathrm{Na}$ seção 2 é apresentado o conceito de gerência de conhecimento, na seção 3 são apresentados alguns modelos de gerência de conhecimento, mostrando o escopo de cada modelo, na seção 4 é descrito o KFM, Knowledge Flow Model, modelo de gerência com foco no fluxo de conhecimento. Na seção 5 será apresentado um estudo de caso sobre a aplicação do KFM em um ambiente de projeto de software. Na seção 6 as conclusões deste trabalho serão apresentadas.

\section{Gerência de Conhecimento}

Gerência de Conhecimento consiste em coletar e armazenar sistematicamente o conhecimento adquirido, compartilhar este conhecimento através de uma memória organizacional e promover o surgimento de novo conhecimento. Neste contexto, conhecimento pode ser definido como informação combinada com experiência, contexto, interpretação e reflexão (Probst).

Tadeu Cruz (Cruz) afirma que quem transforma informação em conhecimento e este gera mais conhecimento, transmitindo para outras pessoas, soube utilizar corretamente o conhecimento.

"A informação se transforma em conhecimento quando utilizada de forma a gerar mais conhecimento. Muitos projetos e processos de software se preocupam com o gerenciamento de conteúdo e artefatos. Os conteúdos são apenas informações que são coletadas e armazenadas para possíveis mudanças. A consulta periódica desses documentos e artefatos é que irá gerar conhecimento, e a análise e aplicação deste conhecimento gerará mais conhecimento.(Cruz)" 
A aplicação de conhecimento no dia a dia em ambientes de trabalho é constante. Sempre há algum tipo de dado, informação ou conhecimento que possa resolver problemas cotidianos. A informação nos dias atuais tem ajudado de diversas maneiras ao crescimento de empresas e instituições em geral. Nunca se teve um fluxo de dados e informação tão grande.

$\mathrm{Na}$ "sociedade do conhecimento" os tradicionais fatores de produção como o trabalho, capital e terra tornam-se secundários (Probst), e o conhecimento passa a ser o recurso mais importante. Probst et al (Probst) reforça essa idéia afirmando que na nova "era da informação" as fontes fundamentais de riqueza são o conhecimento e a comunicação, e não os recursos naturais ou o trabalho físico. Ao que tudo indica, essa transformação é irreversível e incontrolável.

Cruz descreve a sociedade atual como sendo a sociedade do conhecimento. As pessoas precisam de conhecimento para ter uma vida diária que proporcione soluções para os problemas enfrentados. Ao analisar a sociedade, percebe-se a corrida constante em busca do conhecimento (Cruz).

Então, como as pessoas precisavam de informações de conhecimento que já havia sido utilizado, viu-se a necessidade de se administrar esse conhecimento. Cruz (Cruz) ainda define gerência de conhecimento como segue:

"Gerência de Conhecimento, ou Knowledge Manangement (KM), é um conjunto formado por metodologias e tecnologias que têm por finalidade criar condições para identificar, integrar, capturar, recuperar e compartilhar conhecimento existente em qualquer tipo de organização”.

O conceito de gerência de projeto em (CELAPAR) é a aplicação de conhecimento, habilidades, ferramentas, e técnicas às atividades do projeto, de forma a atingir as necessidades e expectativas das partes envolvidas.

Existem vários conjuntos de atividades propostos para a Gerência de Conhecimento. Alguns autores (Falbo et al)afirmam que as atividades básicas da Gerência de Conhecimento são: identificação, aquisição, desenvolvimento, disseminação, uso e preservação de conhecimento. Nessa visão, a memória organizacional está no centro do processo, sendo considerada como um repositório do conhecimento disponível na organização. Para atingir seus objetivos, a Gerência de Conhecimento pode se beneficiar de diversas tecnologias, tais como bancos de dados, Internet, máquinas de busca, dentre outras.

$\mathrm{Na}$ aplicação de Gerência de Conhecimento, alguns conceitos são inseridos, fazendo com que sua utilização seja realizada de forma estruturada. Esses conceitos estão relacionados com os processos essenciais de um modelo de KM (Knowledge Management) e os elementos evolutivos que irão interagir, fazendo com que os modelos de conhecimento sejam utilizados a contento.

Na utilização dos processos essenciais e elementos construtivos de conhecimento, começa-se agrupando e categorizando em termos gerais os problemas encontrados nos ambientes de 
projeto. Isso permite que sejam identificadas diversas atividades que são consideradas essenciais na gerência do conhecimento e que estão todas, muito proximamente, relacionadas. É claro que se podem fazer intervenções em processos essenciais isoladamente, mas isso, inevitavelmente, afetará os demais. Portanto deve-se evitar tentar aperfeiçoar atividades de conhecimento em áreas individuais sem considerar os efeitos mais amplos.

Probst (Probst) classifica os processos essenciais com sendo os seguintes: Aquisição do Conhecimento, Identificação do Conhecimento, Retenção do Conhecimento, Utilização do Conhecimento, Partilha ou Distribuição do Conhecimento e Desenvolvimento do Conhecimento.

Os processos citados têm uma relação de interdependência uma das outras. É importante que quem esta responsável pelo controle destes processos tenha conhecimento para que tais atividades sejam bem aproveitadas e que o conhecimento seja gerado de forma a contribuir para o crescimento intelectual do ambiente de pesquisa.

A Figura 1 mostra a relação entre os processos essenciais descritos.

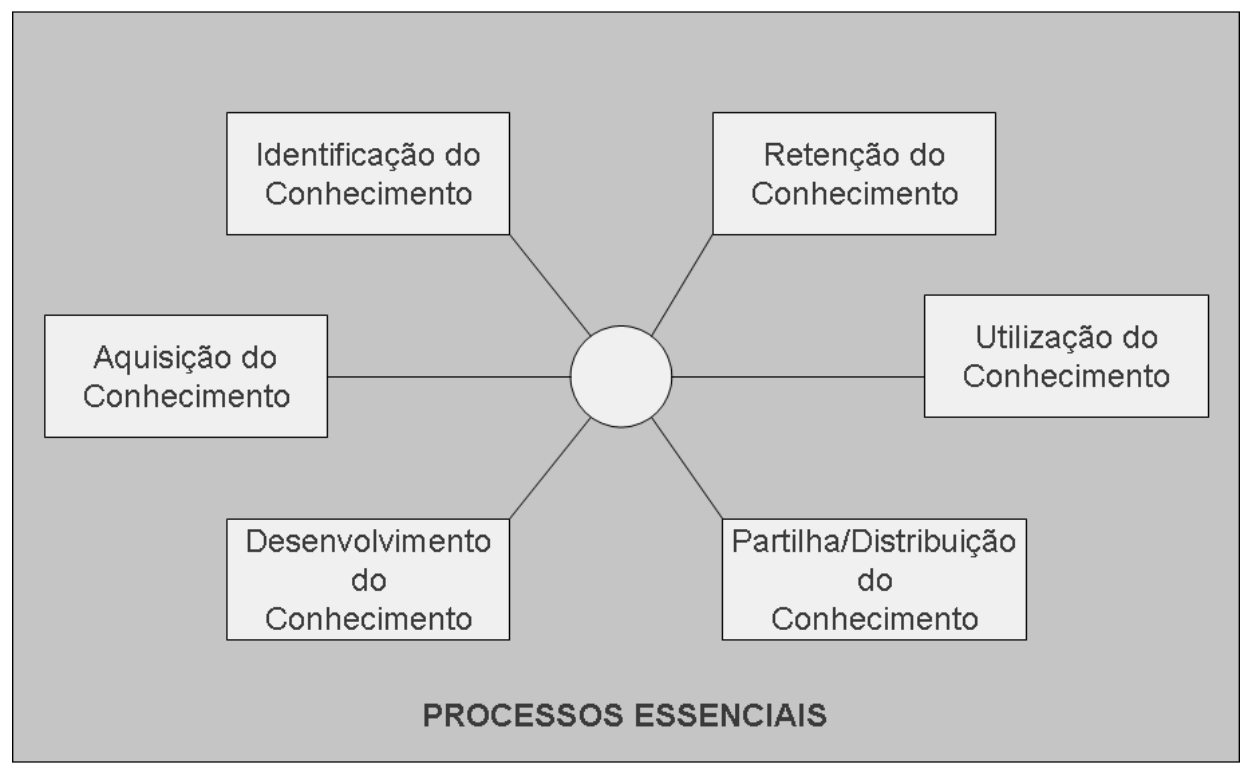

Figura 1. Processos Essenciais

Têm-se como elementos construtivos do conhecimento, os processos essenciais junto com a avaliação do conhecimento e os objetivos estabelecidos para o conhecimento ().

A utilização dos elementos essenciais (Figura 1) no entendimento de gestão ou gerência de conhecimento é base para que os conceitos reais de Knowledge Management sejam aplicados de forma padrão em projetos.

A Figura 2 mostra os elementos construtivos e suas inter-relações lógicas (Probst). 


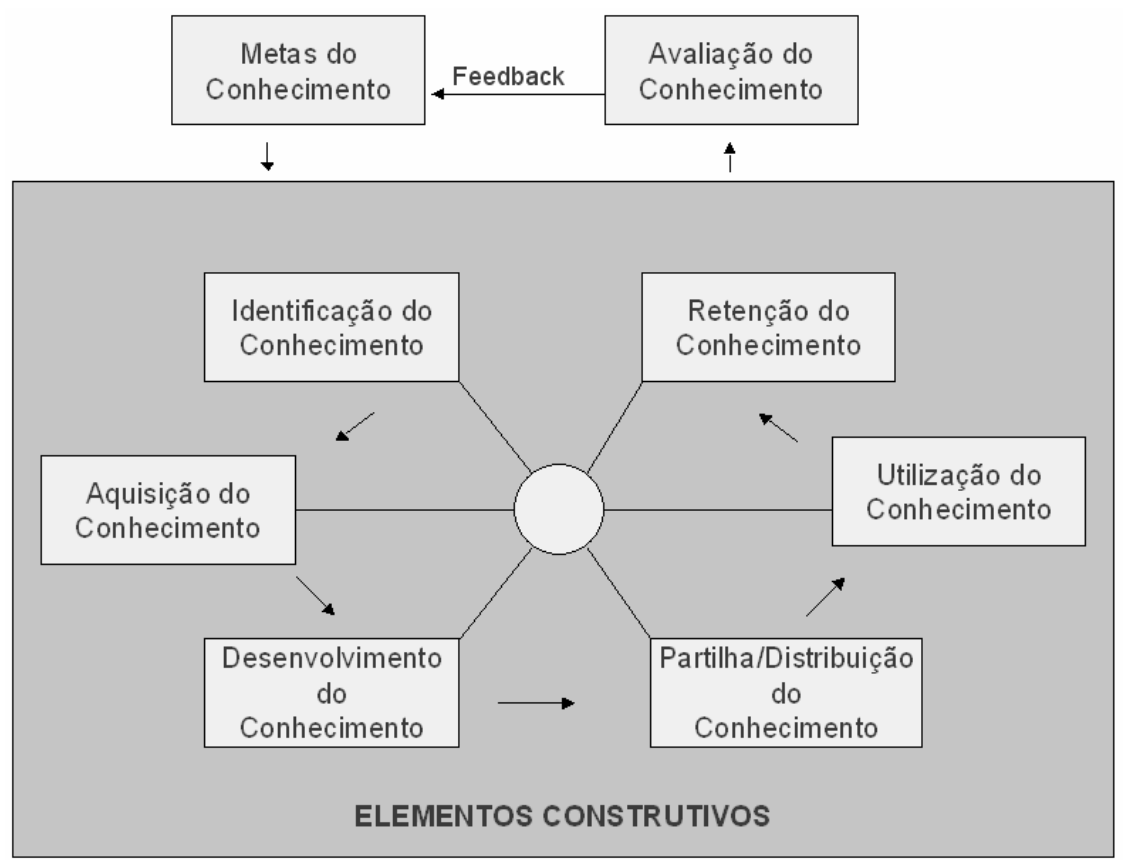

Figura 2. Elementos Construtivos da Gerência do Conhecimento

Em (Probst) é apresentada à avaliação de conhecimento de acordo com os elementos construtivos afirmando que se precisa de métodos para medir os conhecimentos normativos, estratégicos e racionais. A forma como as metas de conhecimento é formulada determina a maneira pela qual podem ser avaliadas. A qualidade das metas, portanto, torna-se aparente no estágio de avaliação, se não ocorrer antes.

Vários modelos de conhecimento são aplicados para uma melhor gerência de conhecimento. Cada organização e projetos distintos utilizam e aplicam estruturas e modelos de gerência de conhecimento diferentes, mas conservando o conceito padrão de gerência de conhecimento(Futami)

Esses conceitos apresentados servem de base para explicar o modelo proposto na fase de desenvolvimento.

\section{Modelos de Gerência de Conhecimento}

Modelos de gerência são criados com o objetivo de aplicar conceitos de conhecimento e gestão de informações e a partir deste ponto especifico mostrar que o modelo está de acordo com os padrões e conceitos base de KM (Futami).

A criação e aplicação de regras, modelos ou estilos arquiteturais para gerência de conhecimento tem sido uma preocupação nos últimos anos. Como foi descrito, o mundo gira em torno de informações e conhecimento que devem ser tratados para serem utilizados para atividades especificas.

Serão apresentados modelos base, desenvolvidos na COPPE/UFRJ (Oliveira et al). Esses modelos abordam a aplicação dos modelos criados em programas de graduação e pósgraduação. Os focos específicos desses modelos são: Término do Projeto, Término da Fase. 
Em um projeto de desenvolvimento de software, as informações que trafegam e o conhecimento existente não deixam de ser coletados. É interessante notar que sempre a constante informação existirá em um projeto de pesquisa e desenvolvimento. Então, como as informações são coletadas, a análise é feita com base na matéria-prima existente, que terá como marco, o final de todo o projeto.

O modelo com foco no término do projeto caracteriza-se por coletar as informações referentes ao ambiente de desenvolvimento ao final no projeto. Essas informações são analisadas e o conhecimento é gerado. $\mathrm{O}$ conhecimento gerado, na utilização deste modelo nunca é utilizado no mesmo projeto de software. Tais informações e conhecimento serão utilizados em projetos subseqüentes (Probst).

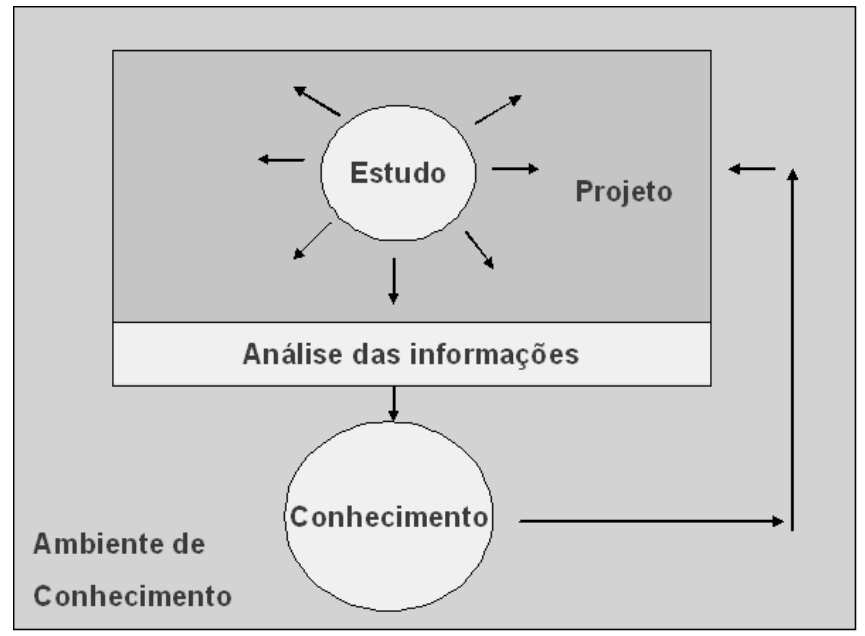

Figura 3. Modelo com Foco no Término do Projeto

A Figura 3 apresenta como funciona o modelo com foco no término do projeto. Existe o ambiente de conhecimento, onde todas as informações circulam. É importante notar nesta figura como ocorre o fluxo de estudo das informações, a análise dessas informações e a evolução do conhecimento gerado. O ponto principal e único da análise da informação é o final do projeto. Os sucessos e dificuldades encontradas durante todo o projeto são analisados somente uma vez por projeto.

Já o modelo com foco no término de fase, as informações geram conhecimento para ser utilizado no próprio projeto. Os marcos de análise das informações, das dificuldades e facilidades são as fases. A cada fase as informações são analisadas e o conhecimento é produzido. O conhecimento na fase final é sempre maior que o conhecimento inicial.

O foco na fase faz com que problemas de implementação da gerência de conhecimento sejam vistos e que o conhecimento melhor produzido.

Esse foi o modelo aplicado no estudo de caso feito por (OLIVEIRA) para verificar e mostrar a importância de utilização de gerência de conhecimento. No artigo base (OLIVEIRA) pode ser encontrado mais detalhes desta aplicação. 


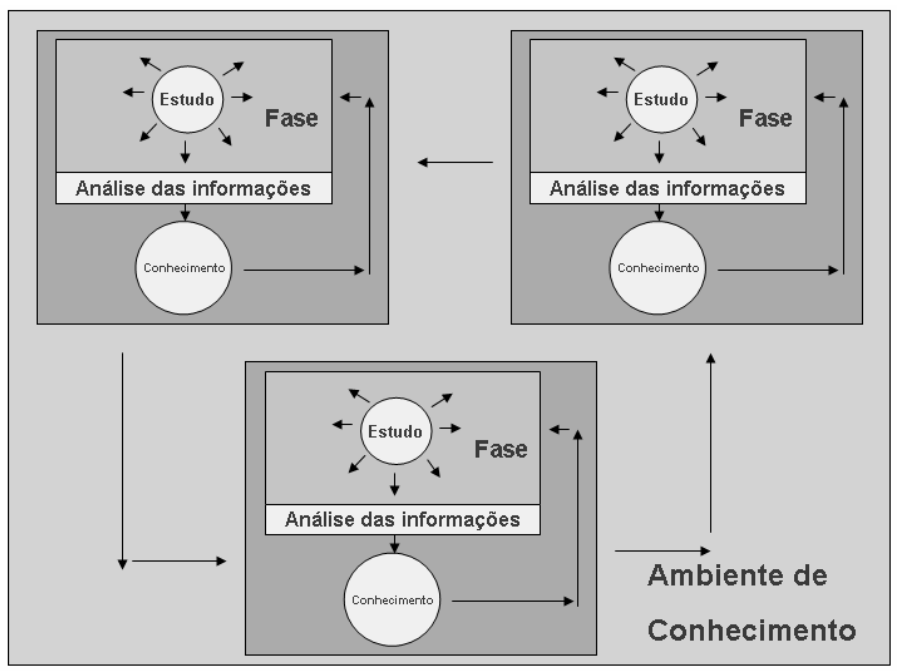

Figura 4. Modelo com Foco no Término da Fase

A Figura 4 mostra como funciona o modelo com foco nas fases de desenvolvimento de um projeto ou processo de software. Nota-se que o conhecimento gerado em uma fase do projeto é utilizado nas fases seguintes, fazendo com que exista um maior domínio das dificuldades e soluções encontradas no decorrer no projeto.

Em projetos de desenvolvimento de software a aplicação de uma norma ou processo de desenvolvimento diminui o número de erros existentes no produto, diminuindo também o tempo de manutenção do produto de software. Como um dos objetivos de processos de software é inserir qualidade ao produto, a aplicação de tais processos ajuda na garantia de qualidade.

\section{KFM - Knowledge Flow Model}

É importante lembrar a preocupação dos modelos supramencionados e suas devidas descrições. Existem tipos de modelos que tentam resolver problemas relacionados a alguma fase de um projeto de pesquisa. E é importante ressaltar que a preocupação com o fluxo de conhecimento não tem sido abordada como um fim, mas como um meio para a concretização e aplicação do modelo.

Pensando nesse contexto, será apresentado um modelo que tem como base a seguinte afirmação: "As informações não entram no início da atividade e nem saem no final da atividade, existe um fluxo de informações que ocorre a todo o momento" (FUTAMI). Os modelos apresentados anteriormente, junto com a afirmação apresentada, servem como base forte para aplicação dessa proposta (modelo) para gerência de conhecimento.

O modelo proposto tem como foco o fluxo de informações e conhecimento existente em um ambiente de projeto e desenvolvimento.

Para melhor identificação do modelo proposto em relação aos modelos apresentados, o modelo proposto será descrito como: Knowledge Flow Model, ou Modelo de Fluxo de Conhecimento, ou ainda descrito pela sigla KFM. Este modelo tem como objetivo propor 
uma melhor forma de coletar informação e conhecimento durante um projeto de desenvolvimento. Além da coleta de conhecimento e informação é interessante que haja uma forma de ter maior proveito desse conhecimento armazenado, para que mais conhecimento seja gerado, em específico o desenvolvimento de software seja um ambiente onde a diferença de conhecimento entre os participantes do projeto seja cada vez menor.

O KFM focaliza no fluxo de informações e como essas informações podem ser coletadas para gerar conhecimento que possa ser utilizado para resolução de algum problema futuro, dentro do escopo do projeto de desenvolvimento que está sendo aplicado o modelo KFM.

Inicialmente, para utilização do KFM, é necessária uma base de conhecimento para armazenamento das informações que serão coletadas no decorrer do projeto. Essa base de conhecimento pode ser abstraída, pois projetos diferentes poderão utilizar base de dados de conhecimento distinta uma das outras.

Definida a base de conhecimento para aplicação do modelo em um projeto, será iniciada a utilização dos conceitos do KFM.

O modelo apresenta partes essenciais. A estrutura, para que o modelo seja conservado, na aplicação em um ambiente de pesquisa deve ser seguida. Projetos que utilizarem uma definição diferente estarão instanciando um novo modelo, onde existem conceitos de fluxo, mas não o foco em fluxo de conhecimento.

A

Figura 5 mostra de forma abstrata a estrutura do modelo:

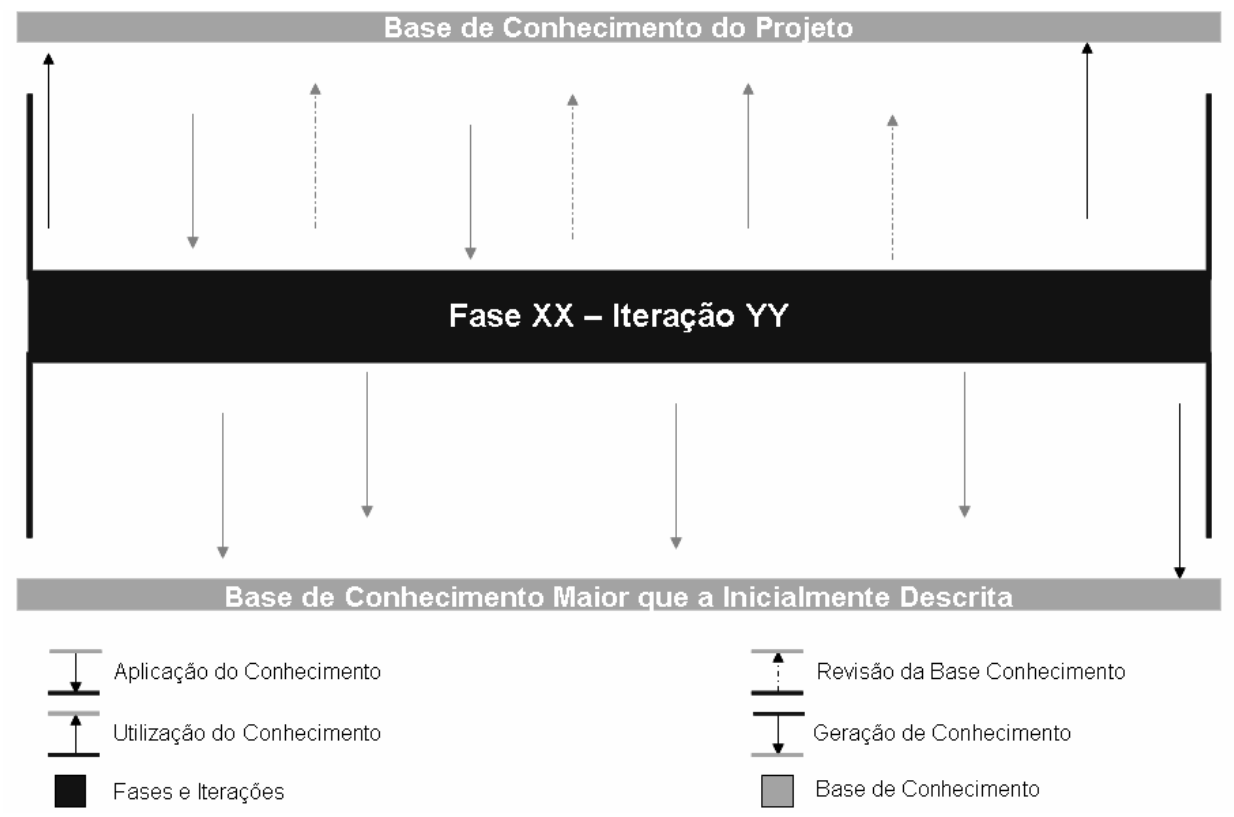

Figura 5. Estrutura do Modelo

Ao iniciar uma fase ou uma iteração, é necessário saber sobre a existência de conhecimento e informações no ambiente de pesquisa e desenvolvimento. Sempre que é necessário conhecer 
ou buscar algum conhecimento é utilizada a estrutura de seta que parte do centro da fase para a parte superior da base de conhecimento.

É importante descrever o significado deste tipo de tarefa, que o modelo proporciona para quem está implementando.

$\mathrm{Na}$

Figura 5 tal tarefa é descrita como "Utilização do Conhecimento". O usuário do modelo faz uma pesquisa para saber como o projeto e suas fases estão estruturadas em relação ao conhecimento e informações existentes.

O marco inicial da aplicação do KFM é conhecer a base de conhecimento, isso se aplica a cada iteração e consequentemente a cada fase. E faz com que as pessoas tenham conhecimento de dificuldades e soluções que problemas antigos provocaram. Deste modo, caso haja uma dificuldade, esta poderá ser resolvida facilmente, pois a base de conhecimento proporciona soluções anteriores, para os tipos de problemas resolvidos; e caso o objetivo seja obter soluções, as pessoas poderão saber como se posicionar diante das dificuldades que as fases e iterações podem apresentar.

A segunda tarefa do modelo KFM, "Aplicação do Conhecimento", mostra a aplicação de informações ou conhecimento para que estes venham a gerar conhecimento. A aplicação do conhecimento é feito de forma a solucionar problemas mais rapidamente, para que a qualidade do produto desenvolvido seja maior e melhor e para que o tempo de produção diminua.

A aplicação do conhecimento consiste em utilizar conceitos existentes na base de dados ou base de conhecimento do modelo e aplicar no próprio projeto, com a meta de gerar conhecimento que será utilizado posteriormente.

A "Revisão da Base de Conhecimento" pode ser utilizada a qualquer momento no projeto de desenvolvimento, não sendo uma tarefa seqüencial do modelo.

A qualquer momento qualquer pessoa pode ir à base de conhecimento e tirar dúvidas existentes ou até mesmo entender o conhecimento que foi gerado no decorrer dos trabalhos passados. Essa tarefa também pode ser uma tarefa que tenha ao final de sua aplicação um conhecimento gerado, pois aplicará as informações colhidas em uma área especifica do projeto e assim aplicar o conceito de conhecimento.

Por último o KFM utiliza a "Geração de Conhecimento". A Geração de Conhecimento é o objetivo principal do modelo. É normal pensar em como gerar conhecimento e ter preocupação com uma gerência que colete informações, dados e conhecimento suficiente para que a base de dados seja povoada e que seja tirado proveito de todo conhecimento gerado. Sendo que esta tarefa do modelo é conseqüência de todas as anteriores junto com a aplicação correta do modelo. 


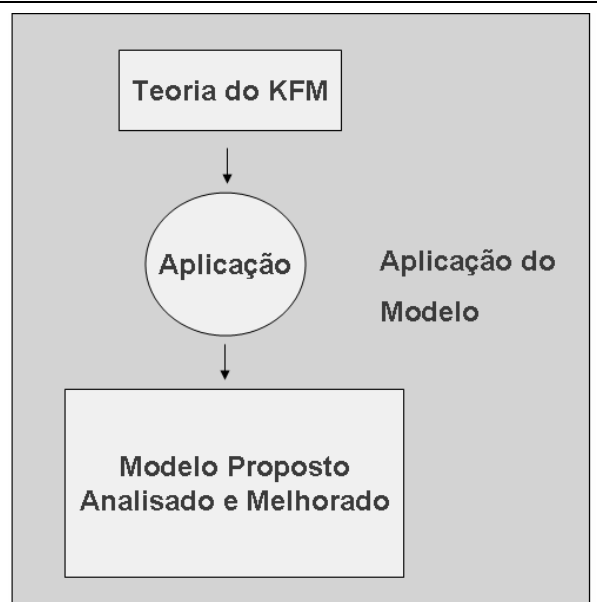

Figura 6. Método de Aplicação do Modelo

A Figura 6 mostra graficamente a atividade de aplicação da abstração teórica com o objetivo de atingir a um modelo e estrutura concreta, que facilite o entendimento teórico e que futuras aplicações do KFM possam ser facilmente utilizadas.

A Figura 6 descreve a transformação dos conceitos de fluxo de conhecimento para o próprio modelo. Primeiramente o modelo de fluxo foi criado de maneira prática e incremental. A cada revisão e aplicação dos conceitos, uma nova visão do modelo era estabelecida.

Na Figura 7 é apresentada a estrutura de fluxo de informação e conhecimento em ambientes de conhecimento. Na parte inferior da figura, os dados existentes no ambiente de produção ou de pesquisa se transformam em informação específica quando inseridos em um contexto próprio. No contexto de desenvolvimento de software, onde o modelo KFM foi criado, tais dados são aplicados com o objetivo de interpretar as informações na área onde o dado esta inserido. A partir daí, as informações são utilizadas de forma a ajudar na continuidade da implementação e desenvolvimento. As informações quando aplicadas em áreas específicas proporcionam conhecimento, fazendo com que a base de conhecimento absorva e que seja aplicada futuramente.

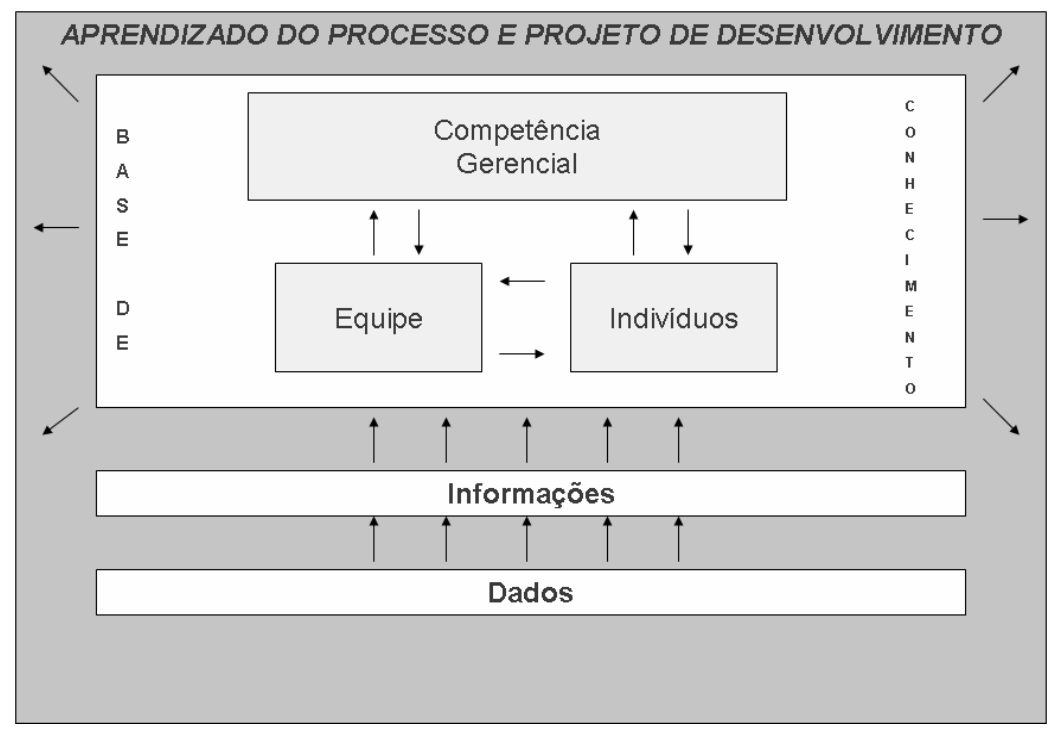

Figura 7. Fluxo de Dados, Informação e Conhecimento 
Para a geração do conhecimento, nota-se, ainda na Figura 7 que além das informações serem inseridas em uma área específica, estas têm que ser manipuladas por alguém. Na parte superior da mesma figura vê-se que existe uma cooperação, indivíduo, equipe e gerência na manipulação dessas informações. O conhecimento só é gerado quando existe essa transformação, pois sem um indivíduo ou um grupo a informação não será utilizada, nem o conhecimento será transformado.

Na Figura 8 as informações são apresentadas para a descrição do modelo. Os números referentes a Figura 8 são respectivamente : 1 - Verificação inicial da base de conhecimento; 2 - Aplicação das experiências anteriores no projeto; 3 - Cadastro de conhecimento referente ao início do projeto; 4 - Análise da base de conhecimento e análise da metodologia; 5 Cadastro de conhecimento referente ao sucesso e dificuldade do uso de informações sobre aplicação e descrição da metodologia; 6 - Aplicação de conhecimento adquirido nas mudanças anteriores no próprio projeto; 7 - Revisão para início da implementação do sistema que aplica o KFM; 8 - Cadastro de informação e conhecimento referente as aplicações de tecnologia em experiências de projetos anteriores; 9 . Consulta à base de conhecimento para continuidade da implementação e resolução de problemas; 10 - Nova revisão da base de conhecimento da inicio dos testes; 11 - Cadastro das informações e conhecimentos referente aos testes do sistema KFM; 12 - Consulta e Revisão Geral da base de dados; 13 - Análise e cadastro do conhecimento referente à revisão e conclusões do projeto.

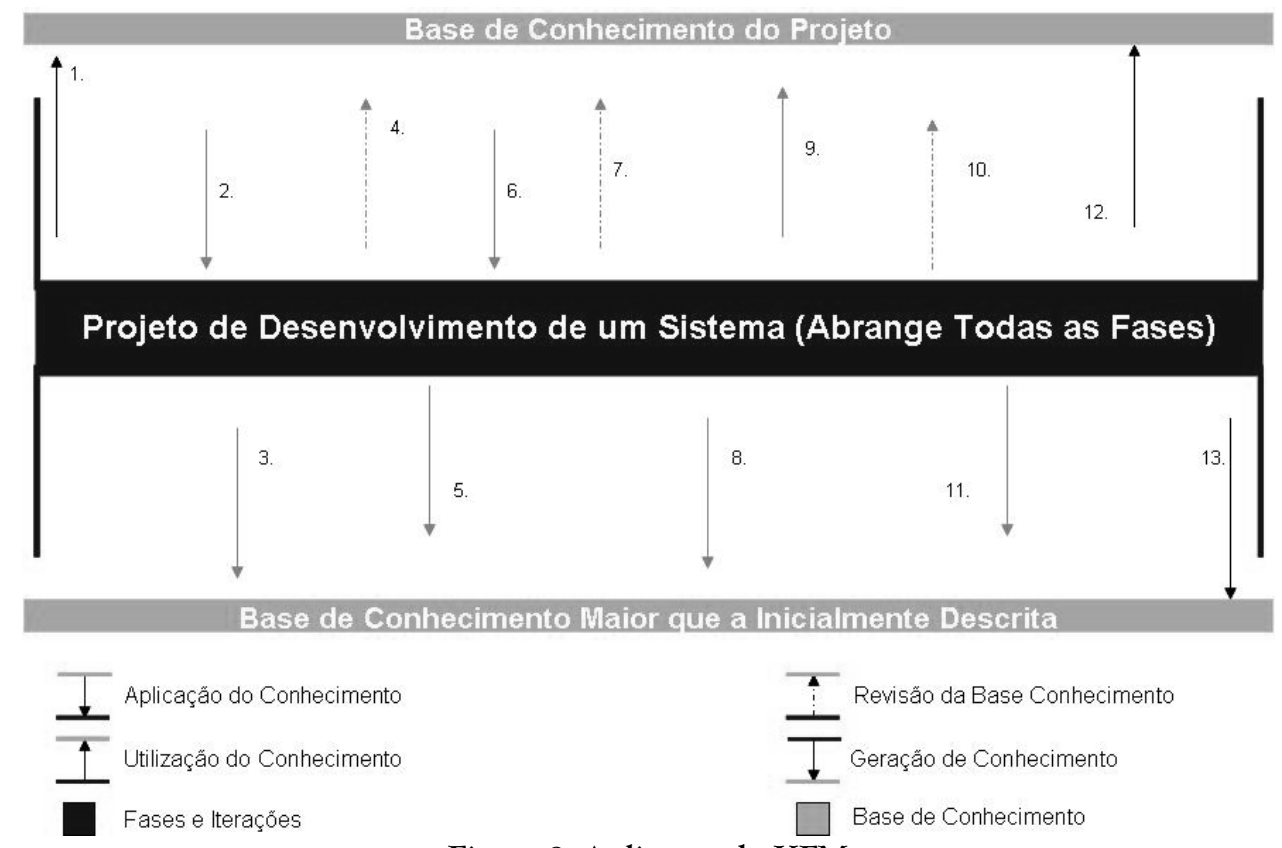

Figura 8. Aplicação do KFM

De acordo com a abstração inicial e os conceitos do modelo de fluxo de conhecimento criado, os resultados produzidos na implementação deste foram de grande importância para a descrição dos conceitos do trabalho. 


\section{Estudo de Caso: Aplicação do KFM do Contexto de Desenvolvimento de Software.}

Utilizando o contexto descrito, a aplicação do KFM foi feita de forma a coletar resultados suficientes para a validação do modelo proposto.

Pensando na forma com que o modelo foi estruturado, com as tarefas estabelecidas, a aplicação seguiu tal descrição com o objetivo de utilizar todos os conceitos.

A Figura 9 apresenta a tela inicial do ambiente desenvolvido para a aplicação do Modelo de Fluxo de Conhecimento. Neste sistema serão utilizadas as funcionalidades do KFM. A seqüência de utilização no sistema é: Inicialmente verifica-se as iterações existentes no projeto ou processo que aplica o modelo KFM, em seguida pode-se adicionar uma informação na iteração, informação esta que poderá ou não se tornar um conhecimento para a equipe. Qualquer membro da equipe que utiliza o KFM poderá ir ao sistema e verificar as informações existentes no banco de conhecimento.

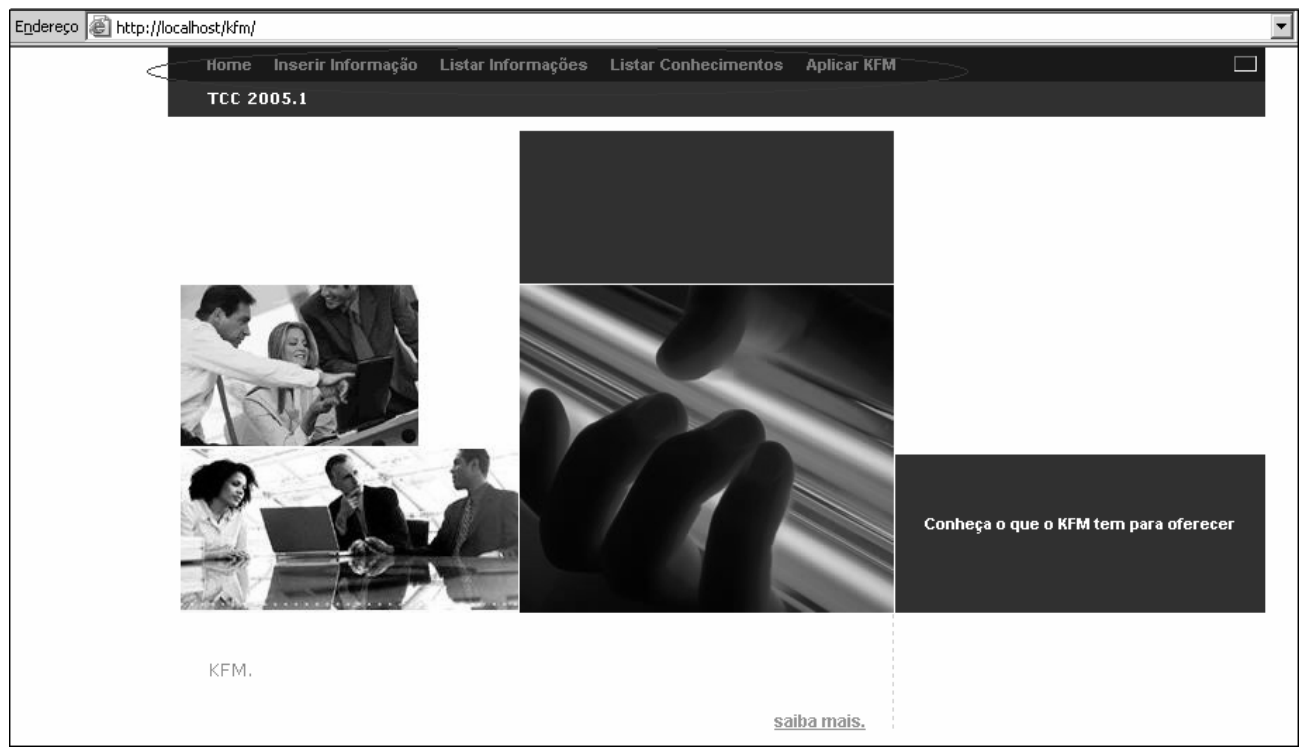

Figura 9. Tela Inicial do Sistema

Na geração do conhecimento a partir do sistema, ao se verificar as informações, o usuário do sistema pode adicionar um conhecimento, pois naquele momento a informação foi utilizada em uma área especifica do projeto de pesquisa ou desenvolvimento. Existem tipos de informação e conhecimento que podem classificar os dados inseridos.

Por fim a partir do conhecimento utilizado podem ser inseridos sucessos e dificuldades do conhecimento. Todos esses dados ficam gravados na base de conhecimento, onde podem ser acessados a qualquer momento para novas atualizações.

A arquitetura escolhida para implementação do sistema foi uma arquitetura que tem como base a arquitetura MVC.

O Modelo MVC, Modelo-Visão-Controlador, representa os objetos ou dados de um aplicativo. A Visão é a representação deste modelo em formato de apresentação para o 
usuário, com interface apropriada para a entrada de informações. O Controlador, por sua vez, programa a interatividade, através do processamento das ações tomadas pelo usuário e atualização do modelo.

O fluxo de interação entre as camadas é cíclico. O usuário indica uma operação através da interface ou visão. Esta operação é processada pelo controlador que altera o modelo conforme a lógica de negócio, retornando ao usuário o novo estado do modelo no formato de uma nova visão. $\mathrm{O}$ usuário prossegue então a sua próxima tarefa, reiniciando o ciclo e assim por diante.

Através dessa arquitetura, o sistema foi desenvolvido. As páginas de requisição e resposta HTML e JSP, são a visão do usuário. O Servlet, as classes JAVA junto com o banco de dados formam o modelo e o controle da arquitetura.

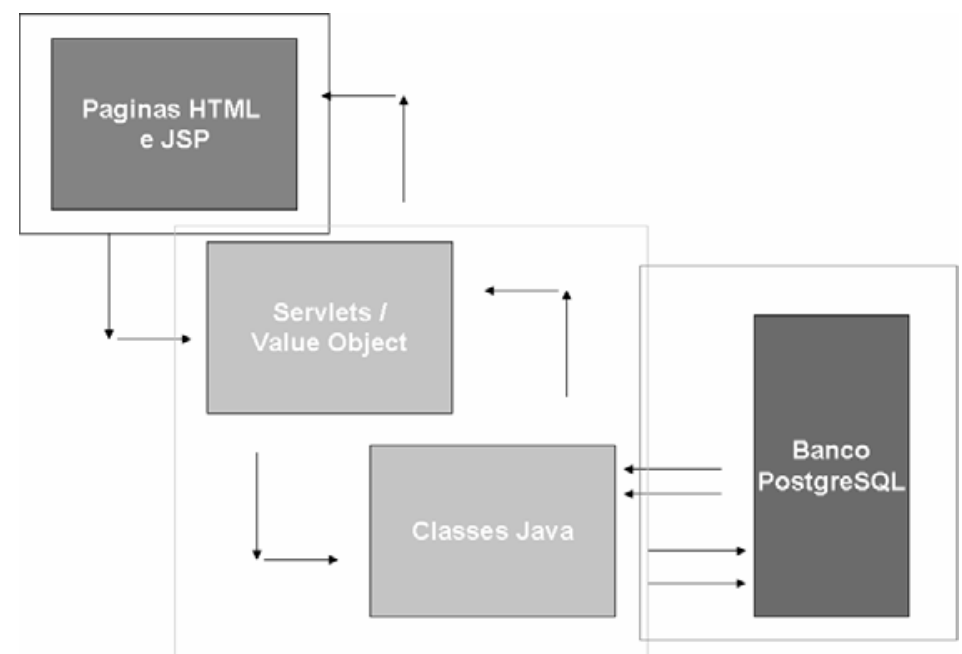

Figura 10. Método de Aplicação do Modelo

A Figura 10 mostra graficamente como o sistema foi desenvolvido.

De acordo com a Figura 10, vê-se a representação da visão através das páginas HTML e JSP que ficam responsável por estabelecer o controle das requisições e receber as respostas no servidor em relação aos dados a serem apresentados. Em seguida tem-se o modelo que fica responsável pela parte intermediária do sistema, fazendo a comunicação do alto nível com o baixo nível - o baixo nível representado, neste caso, pelo controlador que faz a interface com a base de dados. E por fim, o controle é estabelecido pelas classes JAVA responsáveis por tal função como descrito na Figura 10.

O banco de dados PostGreSQL relaciona-se com o modelo MVC de uma forma indireta: recebendo as solicitações e enviando, caso tais solicitações estejam concretas, as respostas necessárias.

Tomando como base experiências anteriores na fase de análise de requisitos, foram coletados os requisitos importantes do sistema a ser desenvolvido. Paralelamente a este procedimento, a base de dados foi sendo povoada com os requisitos estabelecidos, sendo incluído como informações que poderiam ser num momento posterior transformado em conhecimento. 
Durante todo o período de produção, dados e informações eram coletados e conhecimentos foram gerados. Debates sob formas de implementação são levadas em consideração, e inseridas na base de conhecimento, pois é a partir destas informações e das aplicações das informações que o conhecimento é produzido.

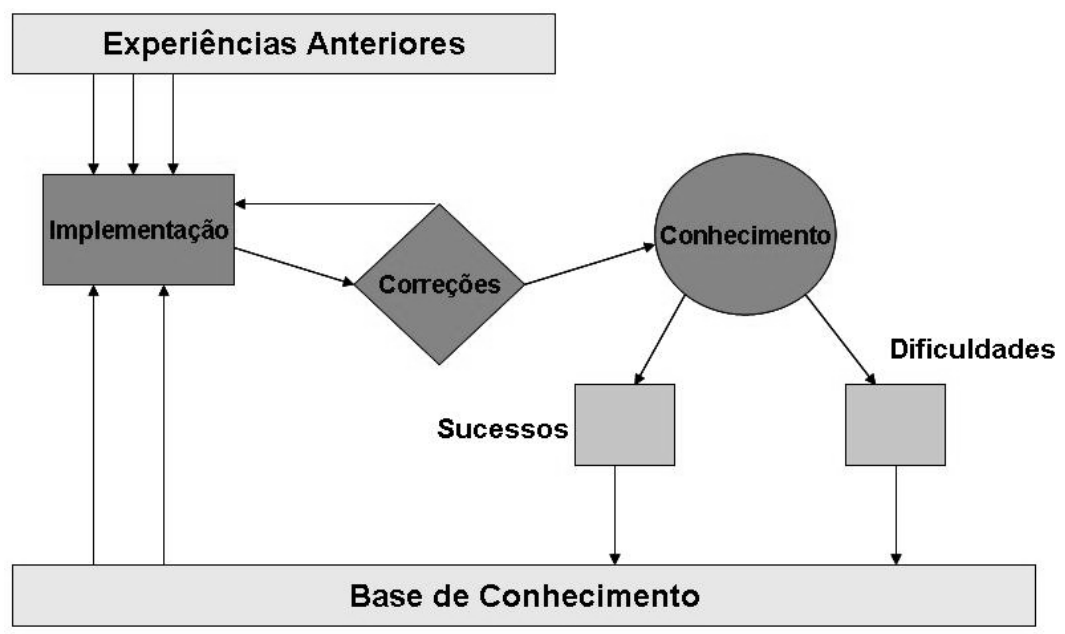

Figura 11. Aplicação do KFM na Implementação

A análise inicial foi feita de acordo com experiências anteriormente aplicadas, partindo do princípio do conhecimento da base de dados. A partir da primeira experiência de análise e modificação, a base de dados foi utilizada, havendo um fluxo de conhecimento retornado aos requisitos iniciais e conseqüentemente na iteração de análise.

A Figura 11 mostra esse fluxo de retorno e aplicação aos requisitos e análise de dados do sistema.

É interessante perceber também na parte superior da Figura 11, que de acordo com as experiências existentes em momentos anteriores da utilização do modelo de fluxo KFM, foram tiradas conclusões que favoreceram ao desenvolvimento da aplicação. É importante que tais experiências sejam inseridas da base de dados na forma de informação para que este seja utilizado em projetos e aplicação futura do KFM.

Especificamente, problemas verificados com conexão com banco de dados, utilização da tecnologia JAVA no desenvolvimento WEB em projetos anteriores foram evitados na aplicação do modelo de fluxo.

Experiências em projetos anteriores ajudaram neste aspecto de dificuldades de tecnologia, já que a base de dados não oferece nenhum retorno em curto prazo. Tais dificuldades e formas de implementação, com seus respectivos sucessos e fracassos foram inseridos na base de conhecimento.

\section{Conclusão}

Gerência de conhecimento é uma área que tem crescido e que existe uma aplicação crescente em ambientes empresariais e acadêmicos. $\mathrm{O}$ estudo dos conceitos ligados à gerência de conhecimento tem feito com que problemas sejam resolvidos, que novas propostas sejam 
feitas e que conclusões de aplicações sejam atingidas. Nesse contexto, aplicar conhecimento novo em um ambiente de projeto é importante para que pesquisas novas surjam e que a capacidade intelectual das pessoas envolvidas no projeto seja proporcional aos conceitos desenvolvidos no ambiente de desenvolvimento.

Contudo, o modelo de fluxo de conhecimento KFM apresentado, implementa os conceitos de gerência de conhecimento junto de conceitos de fluxo de informações. O modelo ajudou no desenvolvimento e na produção constante de conhecimento na implementação de um sistema que gerencia a aplicação do próprio modelo.

\section{Referências}

1. CRUZ, T. Gerência de Conhecimento: Enterprise Content Management. São Paulo - SP, Editora Cobra, 2002.

2. PROBST, G., RAUB, S., ROMHARDT, K.. Gestão de Conhecimento. Os Elementos Construtivos do Sucesso. Porto Alegre - RS, Editora Bookman, 2002.

3. OlIVEIRA, R.F., ROCHA, A.R.C., TRAVASSOS, G.H. Estratégias de Aquisição e Disseminação de Conhecimento para Apoiar o Desenvolvimento de Software com o RUP. COPPE/UFRJ - Programa de Engenharia de Sistemas e Computação.

4. CELEPAR, Companhia de Informática do Paraná. <http://www.pr.gov.br/batebyte/edicoes/2001/bb110/gerência.html> acessado em 30/05/2005

5. MENDES, A. Arquitetura de Software: Desenvolvimento Orientado a Arquitetura. Rio de Janeiro - RJ, Editora Campus, 2002

6. FALBO, R., TOGNERI, D., MENEZES, C., WERNERBACK, B. , ALMEIDA, D., CORTEZ, M. Gerência de Conhecimento na Engenharia de Requisitos

7. FUTAMI, A., VAlENTINA, L., POSSAMAI, O. Um Modelo de Gestão do Conhecimento Para a Melhoria da Qualidade do Produto. Programa de Pós-Graduação em Engenharia de Produção Universidade Federal de Santa Catarina - UFSC 